\title{
Predicting a solar cycle before its onset using a flux transport dynamo model
}

\author{
Arnab Rai Choudhuri ${ }^{1}$ \\ ${ }^{1}$ Department of Physics, Indian Institute of Science, \\ Bangalore - 560012, India \\ email: arnab@iisc.ac.in
}

\begin{abstract}
We begin with a review of the predictions for cycle 24 before its onset. After summarizing the basics of the flux transport dynamo model, we discuss how this model had been used to make a successful prediction of cycle 24, on the assumption that the irregularities of the solar cycle arise due to the fluctuations in the Babcock-Leighton mechanism. We point out that fluctuations in the meridional circulation can be another cause of irregularities in the cycle.
\end{abstract}

Keywords. Sun: activity, Sun: magnetic fields, MHD.

\section{Introduction}

Let us begin with a disclaimer. This review will focus on the physics of predicting solar cycles from dynamo models and will refrain from presenting any detailed prediction for the upcoming cycle 25, which is nowadays becoming a hot topic of research.

Now that we know what the present cycle 24 has been like, let us take a look at the many predictions of cycle 24 before its onset. Pesnell (2008) produced a plot combining all the different predictions of the peak sunspot number of cycle 24. Figure 1 is adopted from this plot, indicating the two predictions based on theoretical dynamo models. The first theoretical prediction by Dikpati et al. (2006) was that cycle 24 would be a very strong cycle, whereas the other prediction by Choudhuri et al. (2007) was that it would be a fairly weak cycle. All the other predictions shown in Figure 1 were based on various precursors and empirical projections. We can see that the predictions covered almost the entire range of possible values of the peak sunspot number from $\approx 40$ to $\approx 190$. The horizontal line indicates the actual peak sunspot number of cycle 24 and was added by us while preparing this presentation. It is clear that Choudhuri et al. (2007) predicted the cycle 24 peak almost correctly.

If several people make several predictions covering the entire possible range, then somebody's prediction has got to come out right! Were Choudhuri et al. (2007) simply the lucky persons whose prediction accidentally turned out to be correct? Or did they get it correct because they figured out the correct physics for making such predictions? We would like to argue that they figured out the correct physics partially, but not fully. Their success in predicting cycle 24 was due to a combination of intuition and luck.

In a classic work, Parker (1955) envisaged that the solar cycle is produced by an oscillation between the toroidal and poloidal magnetic fields of the Sun. Sunspots form out of the toroidal field due to magnetic buoyancy and provide an indication of the strength of the toroidal field. On the other hand, the magnetic fields in the polar regions of the Sun are a manifestation of the poloidal field. We now know that there is truly an oscillation between these two field components. The polar (i.e. poloidal) field becomes strongest around the time when the sunspot number (i.e. toroidal field) has its lowest value and vice versa. Svalgaard et al. (2005) and Schatten (2005), whose predictions for 


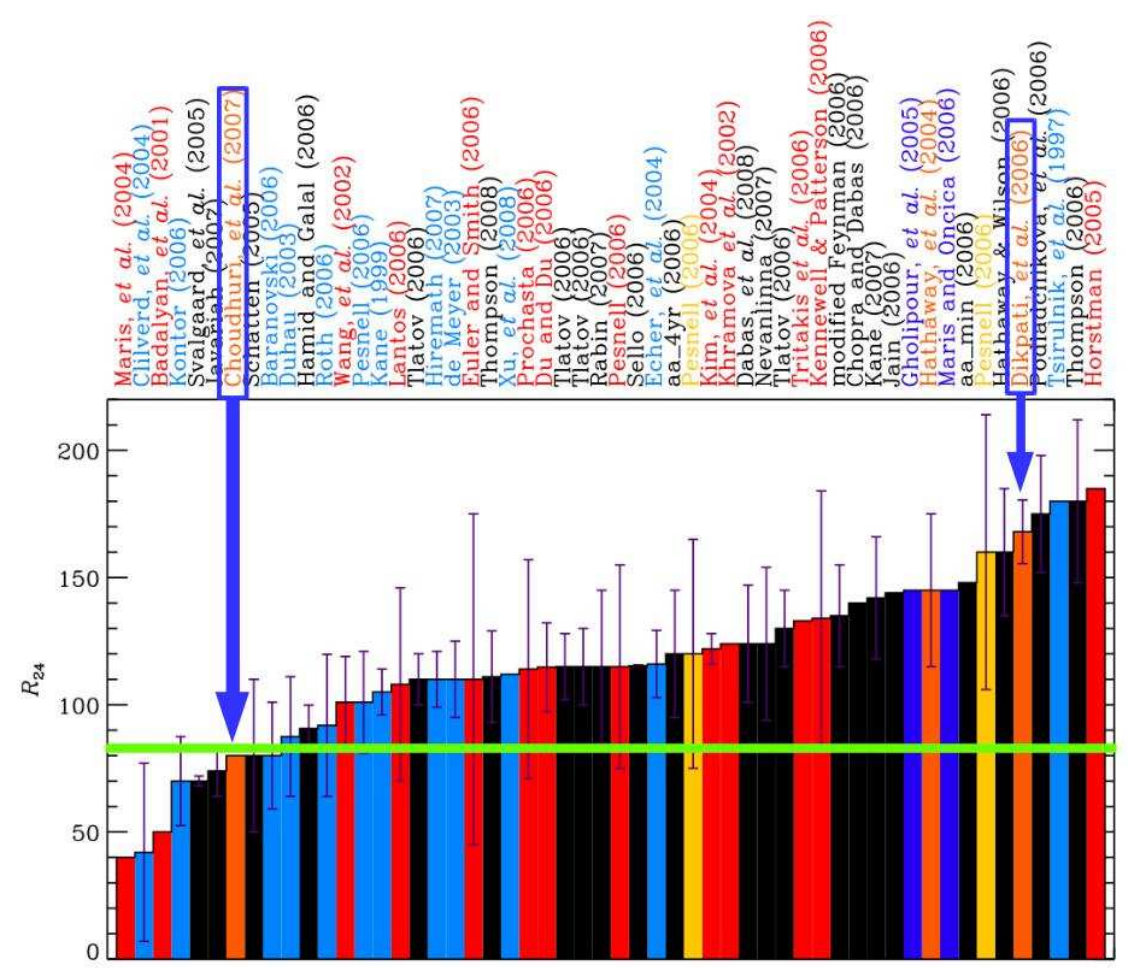

Figure 1. Different predictions of the strength of cycle 24, adopted from Pesnell (2008). The two predictions based on theoretical dynamo models (Dikpati et al. 2006; Choudhuri et al. 2007) are indicated by arrows. The horizontal line added by us indicates the actual peak strength of cycle 24 reached around April 2014.

cycle 24 are included in Figure 1, suggested that the polar field at the beginning of a cycle is a good precursor for the strength of the cycle and used the weak polar field at the beginning of cycle 24 to predict essentially the same low value of the cycle peak that was predicted from the theoretical dynamo calculations of Choudhuri et al. (2007). While discussing the physics of cycle prediction, we need to address the question of why the polar field at the beginning of a cycle acts as such a good precursor of the cycle strength.

\section{Basics of flux transport dynamo model}

The flux transport dynamo model, which started being developed in the 1990s (Wang et al. 1991; Choudhuri et al. 1995; Durney 1995; Dikpati \& Charbonneau 1999; Nandy \& Choudhuri 2002) and has been recently reviewed by several authors (Choudhuri 2011, 2014; Charbonneau 2014; Karak et al. 2014a), has emerged as an attractive theoretical model of the solar cycle. We describe the basics of this model in this Section before getting into the question of cycle prediction in the next Section.

In order to have an oscillation between the toroidal and poloidal magnetic components, we need to have mechanisms to generate each component from the other. The generation of the toroidal component from the poloidal component due to stretching by differential rotation is a straightforward process. To complete the loop, we need a mechanism to generate the poloidal component back from the toroidal component. The flux transport dynamo model assumes that this is achieved by the Babcock-Leighton (BL) mechanism (Babcock 1961; Leighton 1964), in which the toroidal field first gives rise to bipolar 
sunspots which emerge with a tilt due to the action of the Coriolis force (D'Silva \& Choudhuri 1993) and then the poloidal field arises from the decay of these tilted bipolar sunspots (Hazra et al. 2017).

A dynamo model based just on the idea of toroidal field generation by differential rotation and poloidal field generation by the BL mechanism gives a poleward propagating dynamo wave - in accordance with what is called the Parker-Yoshimura sign rule (Parker 1955; Yoshimura 1975). In order to explain the observation that sunspots appear increasingly at lower latitudes with the progress of the solar cycle, we need an extra mechanism to reverse the direction of the dynamo wave and make it propagate equatorward. Choudhuri et al. (1995) realized that this extra mechanism is provided by the meridional circulation (MC) of the Sun, which is observed to be poleward near the surface of the Sun. In order to avoid mass piling up in the polar region, there has to be a subsurface reverse flow towards the equator. The majority of flux transport dynamo models assume an one-cell MC with the equatorward flow at the bottom of the convection zone. However, Hazra et al. (2014) have shown that the flux transport model can work even with a multi-cell MC structure as long as there is an equatorward flow at the bottom of the convection zone. The toroidal field produced there due to the strong differential rotation discovered by helioseismology is advected by this flow equatorward, making sure that sunspots appear at lower latitudes with the progress of the cycle. The poloidal field generated by the BL mechanism near the surface is advected poleward by the poleward MC there - in accordance with observational data.

Perhaps, at this stage, it is a good idea to state clearly what we mean by flux transport dynamo. We would refer to a solar dynamo model as a flux transport dynamo model if it satisfies the following characteristics: (i) the Babcock-Leighton (BL) mechanism for generating the poloidal field at the solar surface is included in the model; and (ii) the meridional circulation (MC) plays an important role by transporting the poloidal field poleward near the surface and the toroidal field equatorward at the bottom of the convection zone. While different authors in the past might have meant slightly different things by flux transport dynamo, we believe that the definition we give here has come to be regarded as the universally accepted definition of flux transport dynamo at the present time.

Detailed theoretical calculations based on the flux transport dynamo model are broadly in agreement with observational data pertaining to the solar cycle. A flux transport dynamo code based on mean field equations tends to give a periodic solution, unless something special is done to make the solution irregular. After our brief summary of the flux transport dynamo model, we come to the question as to what causes irregularities of the cycle and whether an understanding of that will help us in predicting future cycles.

\section{Fluctuations in the Babcock-Leighton (BL) mechanism}

It has been known for some time that fluctuations in the poloidal field generation mechanism can cause irregularities in the cycle (Choudhuri 1992). Choudhuri et al. (2007) suggested that the fluctuations in the BL mechanism would be the main source of irregularities in the dynamo process. It is not difficult to understand how the fluctuations in the BL mechanism may arise. This mechanism depends on the tilts of bipolar sunspot pairs - a pair with a larger tilt making a more significant contribution to the poloidal field. The average tilt of sunspot pairs is given by Joy's law. However, one finds a distribution of tilt angles around this average (Stenflo \& Kosovichev 2012)-presumably caused by the effect of the turbulence in the solar convection zone through which flux tubes rise to form bipolar sunspot pairs (Longcope \& Choudhuri 2002). 


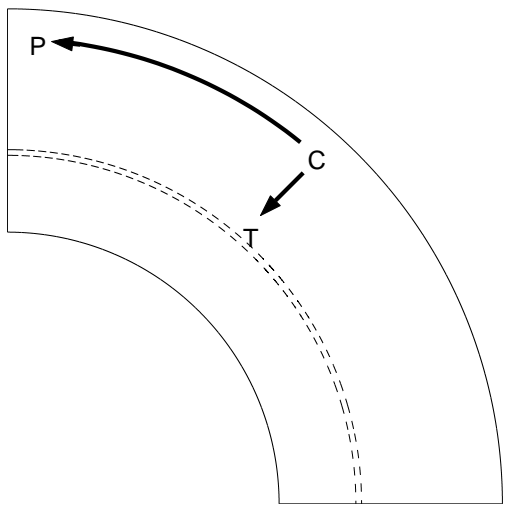

Figure 2. A sketch explaining how the correlation between the polar field at the end of a cycle and the strength of the next cycle arises. From Jiang et al. (2007).

Let us first come to the question how the observed correlation between the polar field at the beginning of a solar cycle and the strength of the cycle arises. Refer to Fig. 2 taken from Jiang et al. (2007), who provided an explanation of this. The BL mechanism produces poloidal field at $\mathrm{C}$ in mid-latitudes near the surface. This poloidal field will simultaneously be advected poleward by the meridional circulation (MC) to produce the polar field at $\mathrm{P}$ at the beginning of the next cycle and will also diffuse to $\mathrm{T}$ at the bottom of the convection zone, where it will act as the seed of the next cycle. If the fluctuations in the $\mathrm{BL}$ mechanism make the poloidal field produced at $\mathrm{C}$ in a cycle stronger than the usual, then both the polar field at $\mathrm{P}$ at the beginning of the next cycle and the seed of the next cycle at $\mathrm{T}$ will be stronger than usual. On the other hand, if the poloidal field produced at $\mathrm{C}$ is weaker than the usual, then the opposite of this will happen. We believe that this is how the correlation between the polar field at the beginning of a cycle and the strength of the cycle arises. It may be pointed out that the turbulent diffusion has to be sufficiently high to ensure that the poloidal field diffuses from $\mathrm{C}$ to $\mathrm{T}$ in time of the order of 5-10 yr, in order to produce the required correlation. Choudhuri et al. (2007) used a value of turbulent diffusivity on the basis of mixing length arguments, which made the correlation come out beautifully. However, Dikpati et al. (2006) used a rather unrealistically low value of turbulent diffusion and the diffusion time across the convection zone in their model is of the order of $200 \mathrm{yr}$. Their model would not give a correlation between the polar field at the beginning of a cycle and the strength of the cycle. It may be mentioned that solar dynamo models with high and low diffusivities have very different characteristics (Jiang et al. 2007; Yeates et al. 2008). There is enough evidence now that models with higher diffusivity are closer to reality. Higher diffusivity helps in explaining such observational features as the dipolar parity (Chatterjee et al. 2004; Hotta \& Yokoyama 2010) and the lack of hemispheric asymmetry (Chatterjee \& Choudhuri 2006; Goel \& Choudhuri 2009).

In order to model actual cycles, one needs to incorporate the actual fluctuations in the BL mechanism into the code. Choudhuri et al. (2007) devised a scheme of figuring out actual fluctuations of the BL mechanism from the observational data of the poloidal fields and then incorporating these in the dynamo code. Since such data are available only from the 1970s, actual cycles could be modelled only from that time. Choudhuri et al. (2007) succeeded in modelling cycles 21-23 reasonably well and cycle 24 was predicted to be a weak cycle. Their prediction of cycle 24 was a robust prediction, since they had incorporated the weakness of the polar field at the beginning of cycle 24 in 
their model and the high diffusivity of their model would make this correlated with the strength of cycle 24 . As we have already pointed out, this prediction has been borne out triumphantly - making this the first successful prediction of a solar cycle based on a theoretical dynamo model in the history of this subject.

\section{Fluctuations in the meridional circulation (MC)}

When Choudhuri et al. (2007) made their prediction, it was not realized that the meridional circulation (MC) probably has occasional large fluctuations and these also may produce irregularities in the solar cycle. These fluctuations are different from the periodic variation of $\mathrm{MC}$ with the solar cycle, presumably due to the Lorentz force of the dynamo-generated magnetic field (Hazra \& Choudhuri 2017). It has been known for some time that the strength of MC determines the period of the dynamo-faster MC making cycles shorter and vice versa (Dikpati \& Charbonneau 1999). Although we have MC data only for about two decades, we have data pertaining to durations of cycles for about two centuries and these data indicate that there have fluctuations in MC with correlation time of the order of a few decades (Karak \& Choudhuri 2011). What kinds of irregularities will the fluctuating MC introduce? Suppose MC has slowed down, making cycles longer. Then diffusion will have more time to act, making cycles weaker. We may thus expect longer cycles to be weaker and shorter cycles stronger. Since a shorter cycle would imply a faster rise time of the cycle, we may expect a correlation between the rise time and the strength of a cycle. Such a correlation is found in the observational data and is known as the Waldmeier effect. Karak \& Choudhuri (2011) gave an explanation of this effect by introducing fluctuations in $\mathrm{MC}$ in their model.

On the basis of such studies, we conclude that there are two important sources of irregularities in the solar cycle - fluctuations in the BL mechanism and fluctuations in MC. Choudhuri et al. (2007) made their prediction of cycle 24 on the basis of the assumption that the irregularities are produced by fluctuations in the BL mechanism alone. Presumably their prediction was so successful because there had not been large fluctuations in $\mathrm{MC}$ in the last few years. Now that we know the fluctuations of MC to be another factor introducing irregularities in solar cycles, we need to develop cycle prediction methods taking this into account. We are presently working on this problem.

It may be mentioned that one big challenge in this field is to develop a theory of grand minima like the Maunder minimum in the 17th century. While it has been shown that grand minima can be induced by fluctuations in the BL mechanism alone (Choudhuri \& Karak 2009) or by fluctuations in MC alone (Karak 2010), we need both of these to develop a comprehensive theory of grand minima (Choudhuri \& Karak 2012; Karak \& Choudhuri 2013).

\section{Conclusion}

Within the last few years, the flux transport dynamo model has emerged as an attractive model for explaining the solar cycle and there is increasing evidence that other solar-like stars also may have similar dynamos working inside them (Karak et al. 2014b; Choudhuri 2017). It is important that we understand how the irregularities in the cycle arise, since such an understanding may enable us to predict a future cycle before its onset. It appears that fluctuations in the BL mechanism and fluctuations in $\mathrm{MC}$ are the two main sources of irregularities in the solar cycle. Before the beginning of cycle 24, the role of MC fluctuations was not generally appreciated. The successful theoretical prediction of Choudhuri et al. (2007) was based on the assumption that irregularities in the solar 
A. R. Choudhuri

cycle are caused only by fluctuations in the BL mechanism. With the realization that MC fluctuations also can introduce additional irregularities, it is necessary to develop prediction methods taking this into account.

Acknowledgments. I thank Gopal Hazra for help in preparing the manuscript. My research is supported by DST through a J.C. Bose Fellowship.

\section{References}

Babcock, H.W. 1961, ApJ, 133, 572

Charbonneau 2014, ARAA, 52, 251

Chatterjee, P. \& Choudhuri, A.R. 2006, Solar Phys., 239, 29

Chatterjee, P., Nandy, D., \& Choudhuri, A.R. 2004, A\&AA, 427, 1019

Choudhuri, A.R. 1992, A\& A, 253, 277

Choudhuri, A.R. 2011, Pramana, 77, 77

Choudhuri, A.R. 2014, Indian J. Phys., 88, 877

Choudhuri, A.R. 2017, Science China Phys. Mech. Astron., 60, 019601

Choudhuri, A.R., Chatterjee, P., \& Jiang, J. 2007, Phys. Rev. Lett., 98, 131103

Choudhuri, A.R., \& Karak, B.B. 2009, Res. Asron. Astrophys., 9, 953

Choudhuri, A.R., \& Karak, B.B. 2012, Phys. Rev. Lett., 109, 171103

Choudhuri, A.R., Schüssler, M., \& Dikpati, M. 1995, A\&A, 303, L29

D’Silva, S., \& Choudhuri, A.R. 1993, A\& $A, 272,621$

Dikpati, M., \& Charbonneau, P. 1999, ApJ, 518, 508

Dikpati, M., de Toma, G., \& Gilman, P.A. 2006, Geophys. Res. Lett., 33, 5102

Durney, B.R. 1995, Solar Phys., 160, 213

Goel, A., \& Choudhuri, A.R. 2009, Res. Asron. Astrophys., 9, 115

Hazra, G., \& Choudhuri, A.R. 2017, MNRAS, 472, 2728

Hazra, G., Choudhuri, A.R., \& Miesch, M.S. 2017, ApJ, 835, 39

Hazra, G., Karak, B.B. \& Choudhuri, A.R. 2014, ApJ, 782, 93

Hotta, H., \& Yokoyama, T. 2010, ApJ, 714, L308

Jiang, J., Chatterjee, P., \& Choudhuri, A.R. 2007, MNRAS, 381, 1527

Karak, B.B. 2010, ApJ, 724, 1021

Karak, B.B., \& Choudhuri, A.R. 2011, MNRAS, 410, 1503

Karak, B.B., \& Choudhuri, A.R. 2013, Res. Asron. Astrophys., 13, 1339

Karak, B.B., Jiang, J., Miesch, M.S., Charbonneau, P., \& Choudhuri, A.R. 2014a, Space Sc. Revs, 186, 561

Karak, B.B., Kitchatinov, L.L., \& Choudhuri, A.R. 2014b, ApJ, 791, 59

Leighton, R.B. 1969, ApJ, 156, 1

Longcope, D., \& Choudhuri, A.R. 2002, Solar Phys., 205, 63

Nandy, D., \& Choudhuri, A.R. 2002, Science, 296, 1671

Parker, E.N. 1955, ApJ, 122, 293

Pesnell, W.D. 2008, Solar Phys., 252, 209

Schatten, K. 2005, Geophys. Res. Lett., 32, L21106

Stenflo, J.O., \& Kosovichev, A.G. 2012, ApJ, 745, 129

Svalgaard, L., Cliver, E.W., \& Kamide, Y. 2005, Geophys. Res. Lett., 32, L01104

Wang, Y.-M., Sheeley, N.R., \& Nash, A.G. 1991, ApJ, 383, 431

Yeates, A.R., Nandy, D., \& Mackay, D.H. 2008, ApJ, 673, 544

Yoshimura, H. 1975, ApJ, 201, 740 